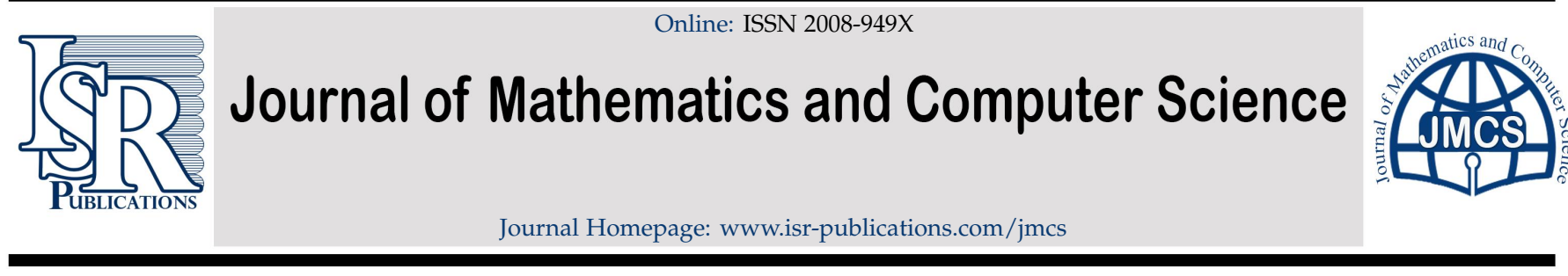

\title{
The category of soft topological spaces and the $T_{0}$-reflection
}

\section{Abdelwaheb Mhemdi \\ Department of Mathematics, Faculty of Sciences and Humanities in Aflaj, Prince Sattam Bin Abdul-Aziz University, Kingdom of Saudi Arabia.}

\begin{abstract}
Soft topological spaces represent the objects of a category named SOFTOP. In this paper, we will study some properties of arrows in SOFTOP. We give also the construction of the $\mathrm{T}_{0}$-reflection of a soft topological space illustrated by other results related to separation axioms.
\end{abstract}

Keywords: Soft set, soft topological spaces, reflective subcategory, separation axioms.

2020 MSC: 06D72, 54A40, 54D10.

(C)2021 All rights reserved.

\section{Introduction}

Since its definition in 1999 by Moldotsov [16], soft sets have been the subject of a lot of researches in different areas of mathematics and computer sciences. Moldotsov and other mathematicians have applied soft sets in game theory, smoothness of functions, Riemann integrations and theory of measurement $[3,13,14,17,18]$.

In 2011, we find the first application of soft sets in topology [2,19]. Shabir and Naz [19] defined for the first time the notion of soft topological spaces which use an initial universe and a fixed set of parameters. Also, separation axioms were studied by different researchers and were the goal of many articles [9, 15].

In this paper, we will see the collection of soft topological spaces as a category named SOFTOP with soft continuous maps as arrows. Soft continuous maps will be studied.

In Section 2, we recall some basic notions and properties of soft sets and soft topological spaces. In Section 3, we will introduce the categorical structure of the collection of soft topological spaces and we will give some category of arrows in this properties. Finally, Section 4 will be devoted to the study of the soft $\mathrm{T}_{0}$-reflection in the category SOFTOP.

\section{Preliminaries}

This section introduces known definitions, notations and properties of soft sets and soft topological spaces.

Definition 2.1 ([16]). Let $X$ be an initial universe containing at least two elements and $A$ a nonempty set of parameters. A soft set over $X$ is a pair $(F, A)$, when $F$ is a map from $A$ to the power set $\mathcal{P}(X)$ of $X$.

Email address: mhemdiabd@gmail .com (Abdelwaheb Mhemdi)

doi: $10.22436 /$ jmcs.022.01.01

Received: 2020-03-05 Revised: 2020-04-09 Accepted: 2020-04-23 
We denote by $\operatorname{SS}(X, A)$ the collection of all soft sets $(F, A)$ over $X$.

Definition $2.2([16])$. Let $(F, A),(G, A) \in S S(X, A)$. We say that $(F, A)$ is a soft subset of $(G, A)$ and we denote $(F, A) \sqsubseteq(G, A)$ if we have $F(a) \subseteq G(a)$ for all $a$ in $A$.

Definition 2.3 ([16]). Let $\left\{\left(F_{i}, A\right): i \in I\right\} \subseteq S S(X, A)$ be a given family of soft sets indexed by an arbitrary set I.

- The soft union of these soft sets is the soft set $(H, A) \in S S(X, A)$ defined by:

$$
\bigsqcup\left\{\left(F_{i}, A\right): i \in I\right\}=(H, A) \text { such that } H(a)=\bigcup_{i \in I} F_{i}(a) \text { for all } a \in A \text {. }
$$

- The soft intersection of these soft sets is the soft set $(H, A) \in S S(X, A)$ defined by:

$$
\sqcap\left\{\left(F_{i}, A\right): i \in I\right\}=(H, A) \text { such that } H(a)=\bigcap_{i \in I} F_{i}(a) \text { for all } a \in A \text {. }
$$

Definition 2.4 ([20]). The soft complement of a given soft set $(F, A) \in S S(X, A)$ is defined by:

$$
(F, A)^{c}=(H, A) \text { such that } H(a)=X \backslash F(a) \text { for all } a \in A .
$$

Notation 2.5 ([16]). In SS (X, A):

- $\mathbf{0}_{\mathrm{A}}$ denotes the soft set $(\mathrm{F}, \mathrm{A}) \in \mathrm{SS}(\mathrm{X}, \mathrm{A})$ such that $\mathrm{F}(\mathrm{a})=\emptyset$ for all $\mathrm{a} \in \mathrm{A}$;

- $\mathbf{1}_{\mathbf{A}}$ denotes the soft set $(F, A) \in S S(X, A)$ such that $F(a)=X$ for all $a \in A$. follows.

The previous definitions and notations prepare us to give the definition of soft topological space as

Definition $2.6([20])$. Let $\tau$ be a family of soft sets in SS $(X, A)$. $\tau$ is called a soft topology on $X$ if it satisfies the following statements:

1. $\mathbf{0}_{\mathbf{A}}, \mathbf{1}_{\mathbf{A}} \in \tau$;

2. if $(F, A),(G, A) \in \tau$, then $(F, A) \sqcap(G, A) \in \tau$;

3. if $\left\{\left(F_{i}, A\right): i \in I\right\} \subseteq \tau$, then $\bigsqcup_{i \in I}\left(F_{i}, A\right) \in \tau$.

The triplet $(X, \tau, A)$ is called a soft topological space. If $(F, A) \in \tau$ then $(F, A)$ is said to be soft open. A soft set $(F, A)$ is said to be soft closed if its complement is soft open and we denote by $\tau^{\mathcal{c}}$ the family of soft closed sets.

According their definitions in topological spaces the separation axioms in soft topological spaces are defined by following.

Definition $2.7([5])$. Let $(X, \tau, A)$ be a soft topological space.

1. We say that $(X, \tau, A)$ is a soft $T_{0}$-space if for every $x \neq y \in X$ there exists $(F, A) \in \tau$, for all $a \in A$, such that $x \in F(a)$ and $y \notin F(a)$ or $x \in F(a)$ and $y \notin F(a)$ which means that the cardinality of $F(a) \cap\{x, y\}=1$.

2. We say that $(X, \tau, A)$ is a soft $T_{1}$-space if for every $x \neq y \in X$ there exists $(F, A) \in \tau$, for all $a \in A$, such that $x \in F(a)$ and $y \notin F(a)$. 


\section{Arrows in the category of soft topological spaces}

This section is devoted to the study of arrows in the category of soft topological spaces.

Definition 3.1 ([16]). Let $X, Y$ be two universes and $A, B$ be two sets of parameters. If $f: X \longrightarrow Y$ and $e: A \longrightarrow B$ are maps then the map $\Phi_{f, e}$ from $S S(X, A)$ to $S S(Y, B)$ is defined by:

$$
\Phi_{f, e}((F, A))=(G, B) \text {, where } G(b)=\bigcup\left\{f(F(a)) \mid a \in e^{-1}(\{b\})\right\} .
$$

Now we give some properties concerning maps presented in the previous definition.

Theorem 3.2. Let $\Phi_{f, e}, \Phi_{g, h}$ be two maps from SS $(\mathrm{X}, \mathrm{A})$ to $\mathrm{SS}(\mathrm{Y}, \mathrm{B})$. Then, the following statements are equivalent:

1. $\Phi_{f, e}=\Phi_{g, h}$;

2. $f=g$ and $e=h$.

Proof. Suppose that $\Phi_{f, e}=\Phi_{g, h}$. On one hand, suppose $e \neq h$. Let $a_{1} \in A$ such that $b_{e}=e\left(a_{1}\right) \neq h\left(a_{1}\right)=$ $b_{h}$. We take the soft set $(F, A)$ defined by $F\left(a_{1}\right)=X$ and $F(a)=\emptyset$ if $a \neq a_{1}$. If $\Phi_{f, e}((F, A))=\left(G_{1}, B\right)$ and $\Phi_{g, h}((F, A))=\left(G_{2}, B\right)$, then $G_{1}\left(b_{e}\right)=f(X) \neq G_{2}\left(b_{e}\right)=\emptyset$ so that $\Phi_{f, e} \neq \Phi_{g, h}$. We deduce that the condition $e=h$ is necessary. On an other hand, suppose that $f \neq g$. Let $x \in X$ such that $f(x) \neq g(x)$. Let $a_{1} \in A$ and consider the soft set $(F, A)$ defined by $F\left(a_{1}\right)=\{x\}$ and $F(a)=\emptyset$ if $a \neq a_{1}$. If $\Phi_{f, e}((F, A))=$ $\left(G_{1}, B\right)$ and $\Phi_{g, e}((F, A))=\left(G_{2}, B\right)$, then $G_{1}\left(e\left(a_{1}\right)\right)=\{f(x)\} \neq G_{2}\left(e\left(a_{1}\right)\right)=\{g(x)\}$ so that $\Phi_{f, e} \neq \Phi_{g, h}$. We deduce that the condition $f=g$ is also necessary.

The converse implication is straightforward.

Theorem 3.3. $\Phi_{f, e}$ is one to one if and only if $\mathrm{f}$ and e are one to one.

Proof. Suppose that $f$ and $e$ are one to one. Let $\left(F_{1}, A\right),\left(F_{2}, A\right) \in S S(X, A)$ such that $\Phi_{f, e}\left(\left(F_{1}, A\right)\right)=$ $\Phi_{f, e}\left(\left(F_{2}, A\right)\right)=(G, B)$. Let $a \in A$. Then, $G(e(a))=f\left(F_{1}(a)\right)=f\left(F_{2}(a)\right)$. Since $f$ is one to one then $F_{1}(a)=F_{2}(a)$. So that $\Phi_{f, e}$ is one to one. Conversely, on one hand, suppose $e$ is not one to one and $a_{1} \neq a_{2} \in A$ such that $e\left(a_{1}\right)=e\left(a_{2}\right)=b$. Let $\left(F_{1}, A\right),\left(F_{2}, A\right) \in S S(X, A)$ defined by $F_{1}\left(a_{1}\right)=F_{2}\left(a_{2}\right)=\emptyset$ and $F_{i}(a)=X$ in all other cases. Then, we have $\Phi_{f, e}\left(\left(F_{1}, A\right)\right)=\Phi_{f, e}\left(\left(F_{2}, A\right)\right)$ so that $\Phi_{f, e}$ is not one to one.

Now, we can assume that $e$ is one to one to get the injectivity of $\Phi_{f, e}$. On another hand, suppose $f$ is not one to one let $x \neq y \in X$ such that $f(x)=f(y)$. Let $a_{1} \in A$ and $\left(F_{1}, A\right),\left(F_{2}, A\right) \in S S(X, A)$ defined by $F_{1}\left(a_{1}\right)=\{x\}, F_{2}\left(a_{1}\right)=\{y\}$ and $F_{1}(a)=F_{2}(a)=X$ if $a \neq a_{1}$. Since $e$ is assumed one to one, we can see that $\Phi_{f, e}\left(\left(F_{1}, A\right)\right)=\Phi_{f, e}\left(\left(F_{2}, A\right)\right)$ which proves that $\Phi_{f, e}$ is not one to one.

Theorem 3.4. $\Phi_{f, e}$ is onto if and only if $f$ and e are onto.

Proof. Suppose that $e$ and $f$ are onto. Let $(G, B) \in S S(Y, B)$. Let $(F, A) \in S S(X, A)$ defined by: $F(a)=$ $f^{-1}(G(b))$ if $e(a)=b$. Since $f$ and $e$ are onto we can verify easily that $\Phi_{f, e}((F, A))=(G, B)$ and then $\Phi_{f, e}$ is onto. Conversely, if $e$ or $f$ are not onto, the $\mathbf{1}_{\mathbf{B}}$ can not be the image of a soft set by $\Phi_{f, e}$ which implies that $\Phi_{f, e}$ can not be onto.

Corollary 3.5. $\Phi_{\mathrm{f}, \mathrm{e}}$ is bijective if and only if $\mathrm{f}$ and e are bijective.

Definition $3.6([4,5])$. Let $(X, \tau, A),(Y, \gamma, B)$ be two soft topological spaces and $f: X \longrightarrow Y, e: A \longrightarrow B$ be two maps. Then, the map $\Phi_{f, e}:(X, \tau, A) \longrightarrow(Y, \gamma, B)$ is said to be soft continuous if for all $(G, B) \in \gamma$ we have $\Phi_{f, e}^{-1}((G, B)) \in \tau$ when $\Phi_{f, e}^{-1}((G, B))=(F, A) \in S S(X, A)$ defined by $F(a)=f^{-1}(G(e(a)))$ for all $a \in A$. 
Proposition $3.7([4])$. Let $(\mathrm{X}, \tau, \mathrm{A}),(\mathrm{Y}, \gamma, \mathrm{B})$ be two soft topological spaces and $\mathrm{f}: \mathrm{X} \longrightarrow \mathrm{Y}, \mathrm{e}: \mathrm{A} \longrightarrow \mathrm{B}$ be two maps. Then, the following statements are equivalent:

1. $\Phi_{f, e}$ is soft continuous;

2. $\Phi_{f, e}^{-1}((G, B)) \in \tau^{c}$ for all $(G, B) \in \gamma^{c}$.

Definition 3.8. Let $X, Y, Z$ be universes, $A, B, C$ be sets of parameters and $f: X \longrightarrow Y, g: Y \longrightarrow Z$, $e: A \longrightarrow B, i: B \longrightarrow C$ be maps. Then the composition of the maps $\Phi_{f, e}: S S(X, A) \longrightarrow S S(Y, B)$ and $\Phi_{g, i}: S S(Y, B) \longrightarrow S S(Z, C)$ is the map $\Phi_{g, i} \circ \Phi_{f, e}: S S(X, A) \longrightarrow S S(Z, C)$ such that $\Phi_{g, i} \circ \Phi_{f, e}=$ $\Phi_{\text {gof, ioe }}$.

Proposition 3.9. If $\Phi_{f, e}$ and $\Phi_{g, i}$ are soft continuous then also $\Phi_{g \circ f, i o e}$ is.

The previous results and definitions present a new category when objects are soft topological spaces and arrows are soft continuous maps $\Phi_{f, e}$. This category will be denoted by SOFTOP. The family of soft

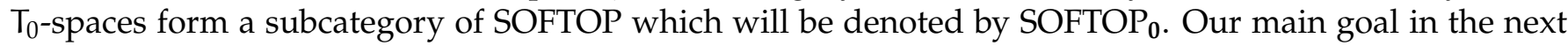
section is to prove that $\mathrm{SOFTOP}_{0}$ is reflective in SOFTOP.

\section{The $\mathrm{T}_{0}$-reflection in SOFTOP}

The construction of the $\mathrm{T}_{0}$-reflection in SOFTOP will be presented in this section.

Using Mac Lane's characterization [12], to show that a full subcategory SOFTOP $_{0}$ is reflective in SOFTOP it is sufficient to prove that: For each $(X, \tau, A) \in \operatorname{SOFTOP}$ there exists an object $(\hat{X}, \hat{\tau}, \hat{A}) \in \operatorname{SOFTOP}_{0}$ and an arrow $\Phi_{h, j}:(X, \tau, A) \longrightarrow(\hat{X}, \hat{\tau}, \hat{A})$ such that for every $(Y, \gamma, B) \in$ SOFTOP $_{0}$ and each arrow $\Phi_{f, e}$ : $(X, \tau, A) \longrightarrow(Y, \gamma, B)$ there exists a unique arrow $\widetilde{\Phi_{f, e}}:(\hat{X}, \hat{\tau}, \hat{A}) \longrightarrow(Y, \gamma, B)$ rendering the following diagram commutative

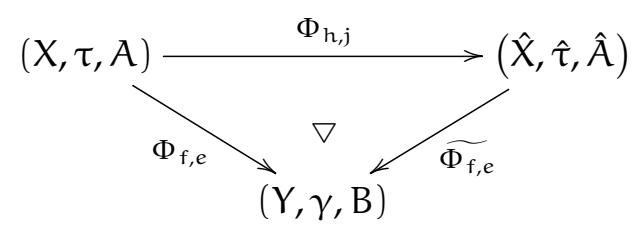

Notation 4.1. Let $(X, \tau, A)$ be a soft topological space. For all $x \in X$ and all $\mathrm{a} \in A$ we denote by $\widetilde{x}^{a}$ the subset of $\mathrm{X}$ defined by:

$$
\widetilde{x}^{a}=\bigcap_{\substack{(F, A) \in \tau^{c} \\ x \in F(a)}} F(a) .
$$

Proposition 4.2. Let $(\mathrm{X}, \tau, \mathrm{A})$ be a soft space. Then, the following statements are equivalent:

1. $(X, \tau, A)$ is a soft $T_{0}$-space;

2. For all $\mathrm{a} \in \mathrm{A}$ we have $\widetilde{x}^{\mathrm{a}}=\widetilde{y}^{\mathrm{a}}$ implies $\mathrm{x}=\mathrm{y}$.

Proof. The proof is Straightforward.

Let $(X, \tau, A)$ is a soft topological space. We define on $X$ the relation $\approx$ by:

$$
\approx \text { by } x \approx y \text { if and only if } \forall a \in A \widetilde{x}^{a}=\widetilde{y}^{a} .
$$


It is clear that $\approx$ is an equivalence relation. We denote by $\mathrm{X} / \approx$ the quotient set and $\mu_{\mathrm{X}}$ the canonical surjection from $X$ onto $X / \approx, x \longmapsto \mu_{X}(x)=\bar{x}$.

Now, for all $(F, A) \in S S(X, A)$ we denote by $(\tilde{F}, A) \in S S(X / \approx, A)$ defined by

$$
\tilde{\mathrm{F}}(\mathrm{a})=\{\bar{x} \mid x \in \mathrm{F}(\mathrm{a})\} .
$$

Let $\tau / \approx$ the subset of $S S(X / \approx, A)$ defined by $\tau / \approx=\{(\tilde{F}, A) \mid(F, A) \in \tau\}$. Clearly, $\tau / \approx$ is a soft topology on $\mathrm{X} / \approx$ and then $(\mathrm{X} / \approx, \tau / \approx, A)$ is a soft topological space. By the construction of the soft topology $\tau / \approx$ the map $\Phi_{\mu_{x}, \mathrm{id}}$ is soft continuous onto map.

Proposition 4.3. $(\mathrm{X} / \approx, \tau / \approx, \mathrm{A})$ is a soft $\mathrm{T}_{0}$-space.

Proof. Let $\bar{x} \neq \bar{y} \in X / \approx$. Then for all $a \in A$ there exists $(F, A) \in \tau$ such that, for example, $x \in F(a)$ and $y \notin F(a)$ which implies that $\bar{x} \in \tilde{F}(a)$ and $\bar{y} \notin \tilde{F}(a)$. So that $(X / \approx, \tau / \approx, A)$ is a soft $T_{0}$-space.

Theorem 4.4. The subcategory SOFTOP 0 of soft $\mathrm{T}_{0}$-spaces is reflective in SOFTOP.

Proof. Let $(Y, \gamma, B)$ be a soft $T_{0}$-space and $\Phi_{f, e}:(X, \tau, A) \longrightarrow(Y, \gamma, B)$ be soft continuous. It is sufficient to prove that there exists a unique soft map $\Phi_{g, h}=\widetilde{\Phi_{f, e}}$ rendering the following diagram commutative.

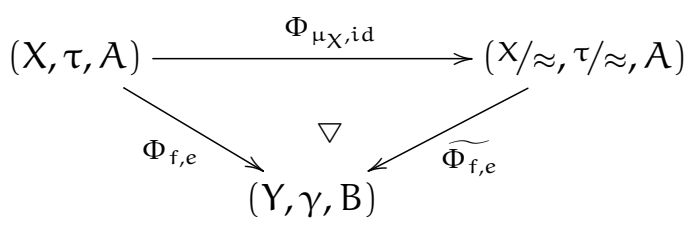

We define $\Phi_{g, h}((\tilde{F}, A))$ to be equal $\Phi_{f, e}((F, A))$ then by Theorem 3.2, g must be defined by $g(\bar{x})=f(x)$ and $e=h$.

Uniqueness: $g$ is well defined: If $f(x) \neq f(y)$ then for all $b \in B$ there exists $\left(G_{b}, B\right) \in \gamma$ such that, for example, $f(x) \in\left(G_{b}, B\right)$ and $f(y) \notin\left(G_{b}, B\right)$. Then for all $a \in A$, there exists $\left(G_{e(a)}, B\right) \in \gamma$ such that, for example, $f(x) \in\left(G_{e(a)}, B\right)$ and $f(y) \notin\left(G_{e(a)}, B\right)$. Since $f$ is soft continuous then $\Phi_{f, e}^{-1}\left(\left(G_{e(a)}, B\right)\right) \in \tau$. So that for all $a \in A$ there exists $\Phi_{f, e}^{-1}\left(\left(G_{e(a)}, B\right)\right) \in \tau$ such that, for example, $x \in \Phi_{f, e}^{-1}\left(\left(G_{e(a)}, B\right)\right)$ and $\mathrm{y} \notin \Phi_{f, e}^{-1}\left(\left(G_{e(a)}, B\right)\right)$ which implies that $\bar{x} \neq \bar{y}$.

$\Phi_{g, h}$ is soft continuous: Let $(G, B) \in \gamma$. We have $\Phi_{\mu x, i d}^{-1}\left(\Phi_{g, h}^{-1}((G, B))\right)=\Phi_{f, e}^{-1}((G, B)) \in \gamma$. Then $\Phi_{g, h}^{-1}((G, B)) \in \tau / \approx$ which implies that $\Phi_{g, h}$ is soft continuous.

conclusion: $(X / \approx, \tau / \approx, A)$ is the soft $T_{0}$-reflection of $(X, \tau, A)$.

Example 4.5. Let $X=\{x, y, z\}, A=\{a, b\}$ and

$$
\tau=\left\{\left(F_{1}, A\right),\left(F_{2}, A\right),\left(F_{3}, A\right),\left(F_{4}, A\right),\left(F_{5}, A\right),\left(F_{6}, A\right)\right\},
$$

where $F_{i}$ are defined by:

- $\mathrm{F}_{1}(\mathrm{a})=\mathrm{F}_{1}(\mathrm{~b})=\emptyset$,

- $F_{2}(a)=F_{2}(b)=X$,

- $F_{3}(a)=\{x, y\}, F_{3}(b)=\{z\}$,

- $F_{4}(a)=\emptyset, F_{4}(b)=X$,

- $\mathrm{F}_{5}(\mathrm{a})=\emptyset, \mathrm{F}_{5}(\mathrm{~b})=\{z\}$, 
- $F_{6}=\{x, y\}, F_{6}(b)=X$.

We can verify that $\tau$ is a soft topology on $X .(X, \tau, A)$ is a soft topological space but it is a not soft $T_{0}$-space because we have $x \neq y \in X$ and $\operatorname{Card}\left(F_{i}(a) \cap\{x, y\}\right) \neq 1$ for all $1 \leqslant i \leqslant 6$.

- $\widetilde{x}^{a}=F_{1}^{c}(a) \cap F_{4}^{c}(a) \cap F_{5}^{c}(a)=X=\widetilde{y}^{a} ;$

- $\widetilde{x}^{b}=F_{1}^{c}(b) \cap F_{3}^{c}(b) \cap F_{5}^{c}(b)=X \cap\{x, y\} \cap\{x, y\}=\{x, y\}=\widetilde{y}^{b}$;

- $\widetilde{z}^{a}=F_{1}^{c}(a) \cap F_{3}^{c}(a) \cap F_{4}^{c}(a) \cap F_{5}^{c}(a) \cap F_{6}^{c}(a)=\{z\}$;

- $\widetilde{z}^{\mathrm{b}}=\mathrm{F}_{1}^{\mathrm{c}}(\mathrm{a})=\mathrm{X}$.

We can see that $x \approx y$ and then $x / \approx=\{\bar{x}, \bar{z}\}$ and $\tau / \approx=\left\{\left(\widetilde{F_{1}}, A\right),\left(\widetilde{F_{2}}, A\right),\left(\widetilde{F_{3}}, A\right),\left(\widetilde{F_{4}}, A\right),\left(\widetilde{F_{5}}, A\right)\right.$, $\left.\left(\widetilde{F}_{6}, A\right)\right\}$ where

- $\widetilde{\mathrm{F}_{1}}(\mathrm{a})=\widetilde{\mathrm{F}_{1}}(\mathrm{~b})=\emptyset$,

- $\widetilde{\mathrm{F}_{2}}(\mathrm{a})=\widetilde{\mathrm{F}_{2}}(\mathrm{~b})=\mathrm{X} / \approx$,

- $\widetilde{\mathrm{F}_{3}}(\mathrm{a})=\{\bar{x}\}, \widetilde{\mathrm{F}_{3}}(\mathrm{~b})=\{\bar{z}\}$,

- $\widetilde{\mathrm{F}_{4}}(\mathrm{a})=\emptyset, \widetilde{\mathrm{F}_{4}}(\mathrm{~b})=\mathrm{x} / \approx$,

- $\widetilde{\mathrm{F}_{5}}(\mathrm{a})=\emptyset, \widetilde{\mathrm{F}_{5}}(\mathrm{~b})=\{\bar{z}\}$,

- $\widetilde{\mathrm{F}}_{6}=\{\overline{\mathrm{x}}\}, \widetilde{\mathrm{F}_{6}}(\mathrm{~b})=\mathrm{X} / \approx$.

$(\mathrm{X} / \approx, \tau / \approx, \mathrm{A})$ is a soft $\mathrm{T}_{0}-$ space because for the two distinct points $\bar{x}, \bar{z}$ of $\mathrm{X} / \approx$ we have $\widetilde{\mathrm{F}_{3}}$ (a) contains $\bar{x}$ and does not contain $\bar{z}$ and $\widetilde{F_{3}}(\mathrm{~b})$ contains $\bar{z}$ and does not contain $\bar{x}$. But $(X / \approx, \tau / \approx, A)$ is not a soft $T_{1}$-space because for all $1 \leqslant i \leqslant 6$ we have $\widetilde{F_{i}}(b)$ can not contain $\bar{x}$ without containing $\bar{z}$.

We know, of course, that the $T_{0}$-reflection of a given soft topological space is a soft $T_{0}$-space. But, this space constructed may satisfies a stronger separation axioms like $T_{1}$. Now, we have to characterize soft topological spaces such that its $T_{0}$-reflection is a soft $T_{1}$-space. Firstly, the following proposition presents an equivalent definition of soft $\mathrm{T}_{1}$-spaces given by Definition 2.7.

Theorem 4.6. Let $(X, \tau, A)$ be a soft space. Then the following statements are equivalents:

1. $(X, \tau, A)$ is a soft $T_{1}$-space;

2. for all $\mathrm{a} \in \mathrm{A}$ and for all $\mathrm{x} \in \mathrm{X}$ we have $\widetilde{x}^{\mathrm{a}}=\{\mathrm{x}\}$.

Proof.

$(1) \Rightarrow(2)$ Suppose that $(X, \tau, A)$ is a soft $T_{1}$-space. Let $a \in A$ and $x, y$ be two different elements of $X$. Since $(X, \tau, A)$ is $T_{1}$ then there exists $(F, A) \in \tau$ such that $y \in F(a)$ and $x \notin F(a)$, so that $y \notin F^{c}(a), x \in F^{c}(a)$ and $\left(F^{c}, A\right)=(F, A)^{c} \in \tau^{c}$. We can deduce that $y \notin \widetilde{x}^{a}$ and then $\widetilde{x}^{a}=\{x\}$.

(2) $\Rightarrow$ (1) Suppose that $\widetilde{x}^{a}=\{x\}$ for every $x \in X$ and every $a \in A$. Let $x \neq y \in X$ and $a \in A$. Since $y \notin \widetilde{x}^{a}$ and $x \notin \widetilde{y}^{a}$ then there exist $\left(F_{1}, A\right),\left(F_{2}, A\right) \in \tau^{c}$ such that $x \in F_{1}(a) \backslash F_{2}(a)$ and $y \in F_{2}(a) \backslash F_{1}(a)$. That is, $\left(F_{1}^{c}, A\right),\left(F_{2}^{c}, A\right) \in \tau, y \in F_{1}^{c}(a) \backslash F_{2}^{c}(a)$ and $x \in F_{2}^{c}(a) \backslash F_{1}^{c}(a)$. This implies that $(X, \tau, A)$ is a soft $\mathrm{T}_{1}$-space.

The next theorem gives a characterization of soft topological spaces whose $T_{0}$-reflections are soft $T_{1}$ space.

Theorem 4.7. Let $(\mathrm{X}, \tau, \mathrm{A})$ be a soft $\mathrm{T}_{1}$-space. Then the following statements are equivalent: 
1. the $\mathrm{T}_{0}$-reflection $(\mathrm{X} / \approx, \tau / \approx, \mathrm{A})$ of $(\mathrm{X}, \tau, \mathrm{A})$ is a soft $\mathrm{T}_{1}$-space;

2. $\forall x \in X, \forall a \in A$ and $\forall(F, A) \in \tau^{c}$ we have:

$$
F(a) \cap \widetilde{x}^{a} \neq \emptyset \Longrightarrow x \in F(a) ;
$$

3. $\forall x \in X, \forall a \in A$ and $\forall(F, A) \in \tau$ we have:

$$
x \in F(a) \Longrightarrow \widetilde{x}^{a} \subseteq F(a) .
$$

Proof.

$(1) \Rightarrow$ (2) Suppose that $(X / \approx, \tau / \approx, A)$ is a soft $T_{1}$-space. Let $y \in F(a) \cap \widetilde{x}^{a}$. By definition of the topology $\tau / \approx$ we have $(\widetilde{F}, A) \in(\tau / \approx)^{c}$ if and only if $(F, A) \in \tau^{c}$. This implies that

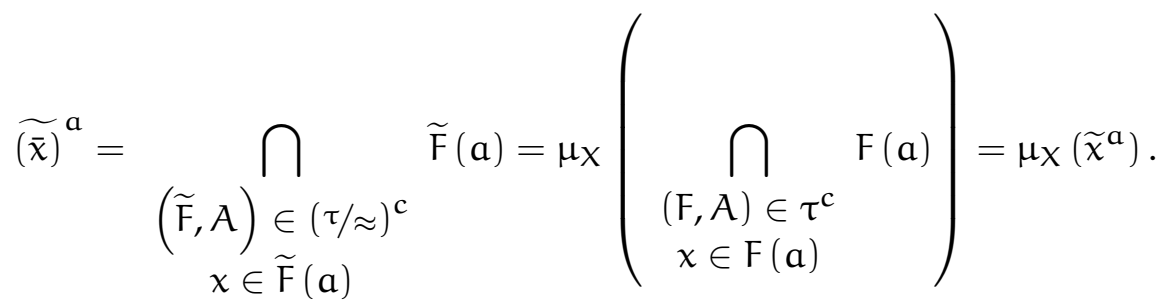

So that $\bar{y} \in \widetilde{(\bar{x})}^{a}=\{\bar{x}\}$ because $(X / \approx, \tau / \approx, A)$ is a soft $T_{1}$-space and using Theorem 4.6. Finally, we can deduce that $\bar{x}=\bar{y}$ and then $x \in F(a)$.

$(2) \Rightarrow(1)$ Let $\bar{x}, \bar{y} \in X / \approx$ and $a \in A$ such that $\bar{y} \in \widetilde{(\bar{x}}^{a}$. It is sufficient to prove that $\bar{x}=\bar{y}$. It is clear that $\widetilde{y}^{a} \subseteq \widetilde{x}^{a}$. Conversely, let $(F, A) \in \tau^{c}$ such that $y \in F(a)$. Now, we have $y \in F(a) \cap \widetilde{x}^{a}$ and using the condition (2) we deduce that $x \in F(a)$ so that $\widetilde{x}^{a} \subseteq \widetilde{y}^{a}$ and finally $\widetilde{y}^{a}=\widetilde{x}^{a}$. This is sufficient to prove that $\widetilde{(\bar{x})}^{a}=\{\bar{x}\}$ for every $\bar{x} \in \mathrm{X} / \approx$. By Theorem $4.6(\mathrm{X} / \approx, \tau / \approx, A)$ is a soft $T_{1}$-space.

(2) $\Leftrightarrow$ (3) Straightforward.

\section{Acknowledgment}

This publication was supported by the Deanship of Scientific Research at Prince Sattam bin Abdulaziz University, Alkharj, Saudi Arabia.

\section{References}

[1] K. Belaid, O. Echi, S. Lazaar, $T_{(\alpha, \beta)}$-spaces and the Wallman compactification, Int. J. Math. Math. Sci., 2004 (2004), 3717-3735.

[2] N. Cagman, S. Karatas, S. Enginoglu, Soft topology, Comput. Math. Appl., 62 (2011), 351-358. 1

[3] D. G. Chen, E. C. C. Tsang, D. S. Yeung, X. Z. Wang, The parameterization reduction of soft sets and its applications, Comput. Math. Appl., 49 (2005), 757-763. 1

[4] D. N. Georgiou, A. C. Megaritis, Soft set theory and topology, Appl. Gen. Topol., 15 (2014), 93-109. 3.6, 3.7

[5] D. N. Georgiou, A. C. Megaritis, V. I. Petropoulos, On soft topological spaces, Appl. Math. Inf. Sci., 7 (2013), 18891901. 2.7, 3.6

[6] A. Grothendieck, Eléments de géométrie algébrique. I: Le langage des schémas, Inst. Hautes Études Sci. Publ. Math., 4 (1960), 228 pages.

[7] A. Grothendieck, J. A. Dieudonné, Eléments de géométrie algébrique, Springer-Verlag, Berlin, (1971).

[8] H. Herrlich, G. Strecker, Categorical topology-Its origins as exemplified by the unfolding of the theory of topological reflections and coreflections before 1971, in: Handbook of the history of general topology, 1997 (1997), 255-341.

[9] S. Hussain, B. Ahmad, Some properties of soft topological spaces, Comput. Math. Appl., 62 (2011), 4058-4067. 1

[10] H.-P. A. Künzi, T. A. Richmond, Ti-ordered reflections, Appl. Gen. Topol., 6 (2005), 207-216.

[11] S. Lazaar, A. Mhemdi, On some properties of $\mathrm{T}_{0}$-ordered reflection, Appl. Gen. Topol., 15 (2014), 43-54.

[12] S. Mac Lane, Categories for the Working Mathematician, Springer-Verlag, New York, (1971). 4

[13] P. K. Maji, R. Biswas, A. R. Roy, Soft set theory, Comput. Math. Appl., 45 (2003), 555-562. 1 
[14] P. K. Maji, A. R. Roy, R. Biswas, An application of soft sets in a decision making problem, Comput. Math. Appl., 44 (2002), 1077-1083. 1

[15] W. K. Min, A note on soft topological spaces, Comput. Math. Appl., 62 (2011), 3524-3528. 1

[16] D. Molodtsov, Soft set theory-first results, Comput. Math. Appl., 37 (1999), 19-31. 1, 2.1, 2.2, 2.3, $2.5,3.1$

[17] D. A. Molodtsov, V. Y. Leonov, D. V. Kovkov, Soft sets technique and its application, Nechetkie Sistemy i Myagkie Vychisleniya, 1 (2006), 8-39. 1

[18] D. Pei, D. Miao, From soft sets to information systems, IEEE Int. Conf. Granular Comput., 2005 (2005), 617-621. 1

[19] M. Shabir, M. Naz, On soft topological spaces, Comput. Math. Appl., 61 (2011), 1786-1799. 1

[20] I. Zorlutuna, M. Akdag, W. K. Min, S. Atmaca, Remarks on soft topological spaces, Ann. Fuzzy Math. Inform., 3 (2012), 171-185. 2.4, 2.6 